\title{
Commissioning the photoinjector of a gamma-ray light source
}

\author{
Han Chen, Lixin Yan, Qili Tian, Zhenyang Lin, Dong Wang, Cheng Cheng, Jiaru Shi, Hao Zha, \\ Yingchao Du, Huaibi Chen, Wenhui Huang, and Chuanxiang Tang \\ Department of Engineering Physics, Tsinghua University, Beijing 100084, China
}

(Received 3 December 2018; published 15 May 2019)

\begin{abstract}
The Xian gamma-ray light source (XGLS) is a proposed novel high brightness gamma-ray source based on inverse Compton scattering (ICS) between the high brightness electron and laser beams. The photoinjector of the accelerator is one of the main components that determines the electron beam quality and related gamma-ray parameters [Phys. Rev. Accel. Beams 21, 030701 (2018); 20, 080701 (2017); $\mathbf{8}, 100702$ (2005)]. In order to reduce the risk of this project, a photoinjector including a laser-driven photocathode rf gun, two S-band tubes, and a diagnostic section was constructed and installed from 2016, serving as a pilot plant for the development of the key technologies for the projects. The injector is designed to deliver high quality electron bunches of typically $500 \mathrm{pC}$ charge and $\sim 120 \mathrm{MeV}$ energy with low emittance and jitter at $10 \mathrm{~Hz}$ repetition. Initial system commissioning with an electron beam was finished in the end of 2017, with a goal of a stable $0.6-0.7 \mathrm{~mm}$ mrad emittance in a 500-pC bunch clearly demonstrated. In this paper, we report the experimental results and experience obtained in the commissioning, including rf gun, drive laser system, timing and synchronization system, and beam diagnostics.
\end{abstract}

DOI: 10.1103/PhysRevAccelBeams.22.053403

\section{INTRODUCTION}

High intensity x-ray light sources based on free electron laser (FEL) and synchrotron accelerators have already set off a wave of exploring the structure and dynamics of atomic and molecular systems around the world [1-9]. However, high intensity quasimonochromatic dedicated gamma-ray light sources are still rare till now. Gammaray sources based on inverse Compton scattering (ICS) are currently the most feasible solution [10]. Since in such sources the incident photons are boosted to high energy proportional to the square of electron beam energy, it is easy to generate high energy gamma-ray beam with much lower beam energy than FEL and synchrotron light source. For example, to generate $1 \mathrm{MeV}$ gamma-ray photon with $800 \mathrm{~nm}$ laser, the required beam energy is about $200 \mathrm{MeV}$, which makes such sources very compact. Besides, gammaray source based on ICS has the feature of high intensity, quasi-monochromatic and good collimation. Dedicated gamma-ray sources based on ICS are being proposed and constructed at many labs worldwide [11-13], and thus the applications of high intensity quasimonochromatic gamma-ray are also being researched and excavated, such as nuclear resonance fluorescence (NRF) imaging, nuclear reaction research and so on $[11,12]$.

Published by the American Physical Society under the terms of the Creative Commons Attribution 4.0 International license. Further distribution of this work must maintain attribution to the author(s) and the published article's title, journal citation, and DOI.
Recently, we start to propose and design the Xian gamma-ray source (XGLS) based on ICS at accelerator laboratory in Tsinghua University, which will deliver 0.1-3 MeV energy tunable monochromatic gamma-ray light of up to $10^{8}$ photons yields per pulse at a repetition of $10 \mathrm{~Hz}$. This implies it requires high quality electron and laser beam at the interaction point. In design, stable 500-pC bunches would be accelerated to about $360 \mathrm{MeV}$ and have the emittance of less than $0.9 \mathrm{~mm}$ mrad. This requirement would be met by careful and systemic design and construction of the photoinjector based accelerator $[14,15]$. In order to reduce the project risk, the photoinjector was constructed and installed from 2016 first to serve as the pilot plant and test bed of the key technologies and components. The main design and typically measured parameters of the photoinjector in XGLS are listed in Table I. Initial commissioning with the first electron beam

TABLE I. Main design and typically measured parameters of the photoinjector in XGLS.

\begin{tabular}{lcc}
\hline \hline Parameter & Design & Measured \\
\hline Bunch charge (nC) & 0.5 & 0.5 \\
Final beam energy (MeV) & $\sim 120$ & $\sim 120$ \\
Energy jitter & $0.1 \%$ & $0.1 \%$ \\
Bunch length (FWHM, ps) & $\sim 10$ & 10.1 \\
Energy spread (rms, \%) & $\leq 0.14$ & 0.18 \\
Projected norm emittance & $\leq 0.9$ & $0.6-0.7$ \\
$\quad$ (mm mrad) & & (in the best case) \\
Repetition rate (Hz) & 10 & 10 \\
\hline \hline
\end{tabular}


was finished in the end of 2017. The measured beam emittance is about $0.6-0.7 \mathrm{~mm}$ mrad in a $500-\mathrm{pC}$ bunch, which clearly demonstrated the goal of the project.

In this paper, we report the first beam results and experience obtained in the commissioning, including photocathode rf gun, drive laser system, timing and synchronization, and beam diagnostics. This main body is organized as follow: in Sec. II, we will present the description of the main components in the photoinjector, including the main subsystems such as rf gun, drive laser system, and timing and synchronization system. In Sec. III, we give some commissioning results of the photocathode rf gun. In Sec. IV, the result of emittance optimization will be presented. And at last a summary and future perspective will be presented.

\section{DESCRIPTION OF THE MAIN COMPONENTS}

\section{A. Overview}

The injector adopts a common layout of a normal conducting S-band $(2.856 \mathrm{GHz})$ photoinjector [8], consisting of a laser-driven photocathode $\mathrm{rf}$ gun, two S-band tubes, and a diagnostic section. The rf gun and two S-band tubes are powered by three sets of modulators and S-band klystrons, respectively. Figure 1 shows the major components of the photoinjector with a total length of about 15 meters. The injector begins with a S-band photocathode rf gun, followed by two three-meters SLAC S-band accelerating tubes. It produces a single electron bunch typically with an energy of $\sim 120 \mathrm{MeV}$ and $0.5 \mathrm{nC}$ of bunch charge at a $10 \mathrm{~Hz}$ repetition rate. Then this beam is transported into a beam diagnostic section, consisting of matching and scanning quadrupoles, an energy analysis dipole magnet, and several beam profile monitors with Yttrium Aluminum Garnet (YAG) and optical transition radiation (OTR) screens. Beam diagnostics for charge and position are located among the beam line, including an integrating current transformer (ICT) for bunch charge measurement, SBPMs (strip-line beam position monitors) and CBPMs (cavity beam position monitors) for beam position measurement. Drive laser system is located in a clean room beside the injector hall and it can deliver $266 \mathrm{~nm}$ ultraviolet (UV) laser pulses with $\sim 10$ ps FWHM duration and variable transverse spot sizes at a $10 \mathrm{~Hz}$ repetition rate.

\section{B. Photocathode rf gun}

The gun installed in the photoinjector is one of the Tsinghua University third-type rf gun which have been demonstrated to have high performance and to generate high quality electron beam on beam lines of different facilities [16-19]. This gun was developed based on the conventional BNL/SLAC/UCLA/KEK type gun $[20,21]$ and improvements were made to improve the performances $[22,23]$. The gun geometry profile was modified to reduce the peak electric field on the iris, increase the quality factor of the cavity and increase the mode separation of the 0 -mode and pi-mode. The gasket between the cathode plate and the first cell was removed and they were brazed directly to reduce the rf breakdown possibility. The simple four ports scheme with nonsymmetric vacuum port was used to eliminate the dipole and quadrupole field. The cathode was cleaned with dry ice after the brazing to reduce the dark current. More detail information about the gun development is introduced in Ref. [23].

\section{Drive laser system}

The drive laser was manufactured by Coherent Corporation and is a frequency tripled, chirped-pulse amplification fs laser system based on Ti:sapphire. It can deliver $266 \mathrm{~nm}$ UV laser with $~ 100$ fs FWHM duration and $\sim 1.5 \mathrm{~mJ}$ maximum energy per pulse at $10 \mathrm{~Hz}$. The laser energy jitter is far less than $1 \%$. The spatial and temporal shapes of this laser are both Gaussian distribution. While in order to reduce the nonlinear space charge effect on beam emittance, the laser pulse should have a uniform distribution with hard edges in space and time [24-27]. Therefore, a spatiotemporal reshaping is required.

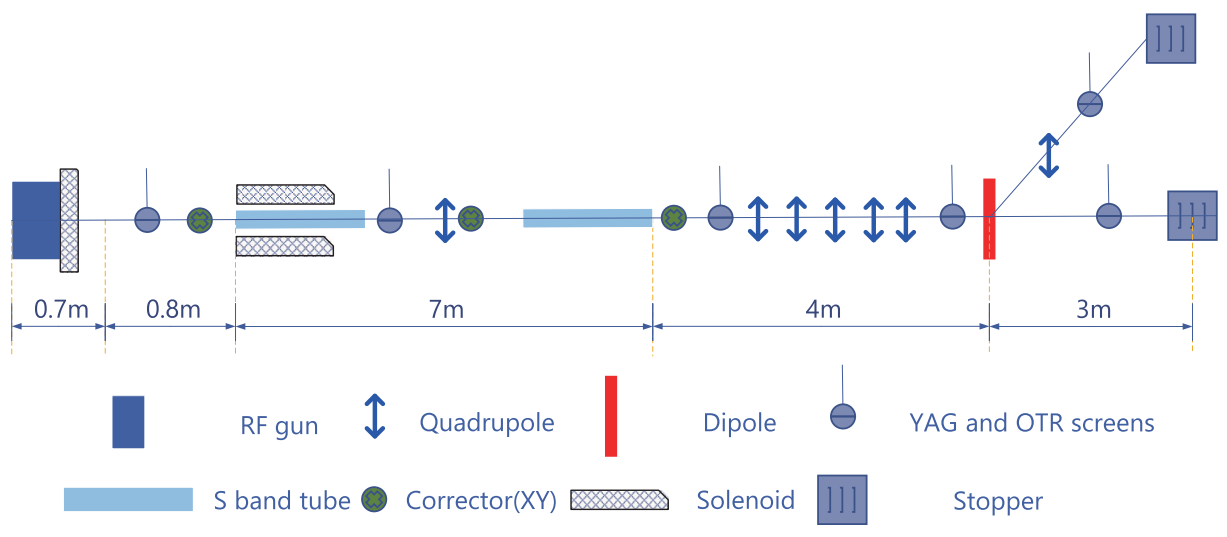

FIG. 1. Layout of the XGLS photoinjector. 


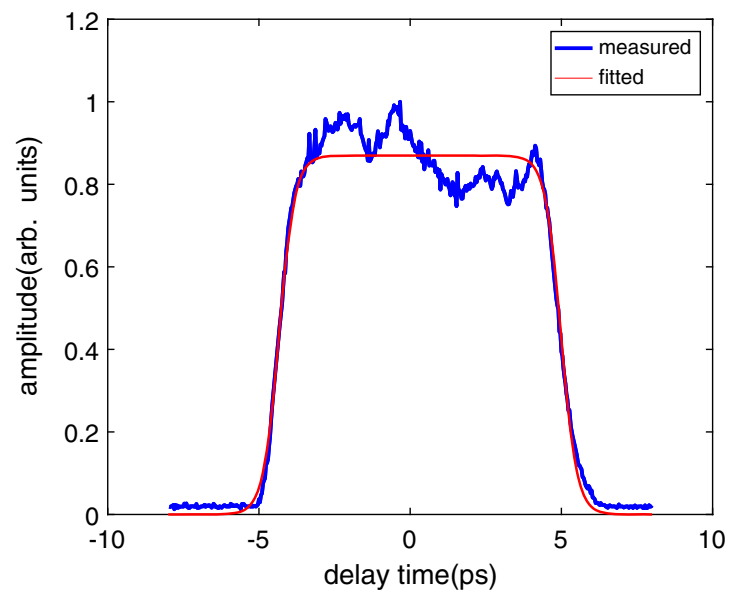

FIG. 2. Measured and fitted temporal profile of the UV laser.

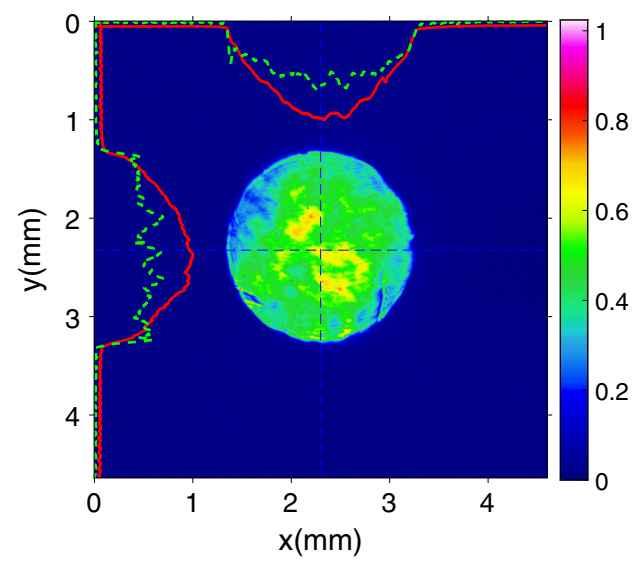

FIG. 3. Transverse profile of UV laser when the BSA is $2 \mathrm{~mm}$. The red solid lines are horizontal and vertical projections. The green dotted lines are the distributions at the blue dashed line positions.

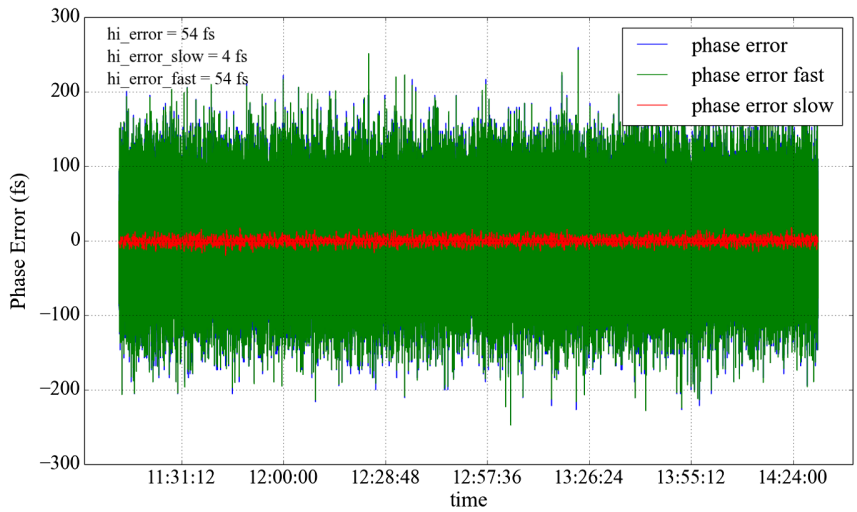

For the temporal shaping, pulse stacking method based on birefringent crystal $\alpha$-BBO is used in the drive laser system [28,29]. We use five pieces of $\alpha$-BBO crystal to separate a input UV laser pulse into 32 subpulses with appropriate time intervals and 32 stacked subpulses form a temporally quasiflat-top laser pulse suitable for cathode driving. The thickness of the five $\alpha$-BBO crystals used for stacking are $5.92 \mathrm{~mm}, 2.96 \mathrm{~mm}, 1.48 \mathrm{~mm}, 0.74 \mathrm{~mm}$, $0.37 \mathrm{~mm}$ respectively. We measured the temporal distribution of the UV laser with a cross-correlation method [30]. We used a bunch of $800 \mathrm{~nm}$ infrared (IR) laser with $100 \mathrm{fs}$ FWHM duration to make difference frequency with the UV laser in a difference frequency crystal, generating the $400 \mathrm{~nm}$ signal light. We scanned the time delay between the IR and UV laser and recorded the change in the signal light intensity with an ultrafast photoelectric detector. Then the temporal distribution of the UV laser was inferred as the blue curve shown in Fig. 2. The red curve in Fig. 2 is the fitting curve with a flat-top distribution and $R^{2}$ is 0.9858 . FWHM duration of the UV laser is $9.32 \mathrm{ps}$, and the rise and fall time between $10 \%$ and $90 \%$ peak is 0.96 ps. The transmission length of the UV laser from the laser system exit to the photocathode is more than $10 \mathrm{~m}$. In order to ensure the uniformity of the spatial distribution of the laser beam onto the photocathode, the so-called relay-imaging technique is used in the optical transport line [31,32]. In the drive laser system, the excellent near-field distribution is maintained by two stage relay-imaging systems, which effectively overcomes the diffraction effect. Each relayimaging system consists of two focusing lenses. A variable laser beam shaping aperture(BSA) is placed at the image plane of first relay-imaging system. The laser spot size on the photocathode can be controlled by changing the diameter of BSA, including $1.2 \mathrm{~mm}, 1.4 \mathrm{~mm}, 1.6 \mathrm{~mm}$, $1.8 \mathrm{~mm}, 2 \mathrm{~mm}$, and $2.3 \mathrm{~mm}$. Figure 3 shows the transverse profile of UV laser at the virtual cathode plane when the BSA is $2 \mathrm{~mm}$.

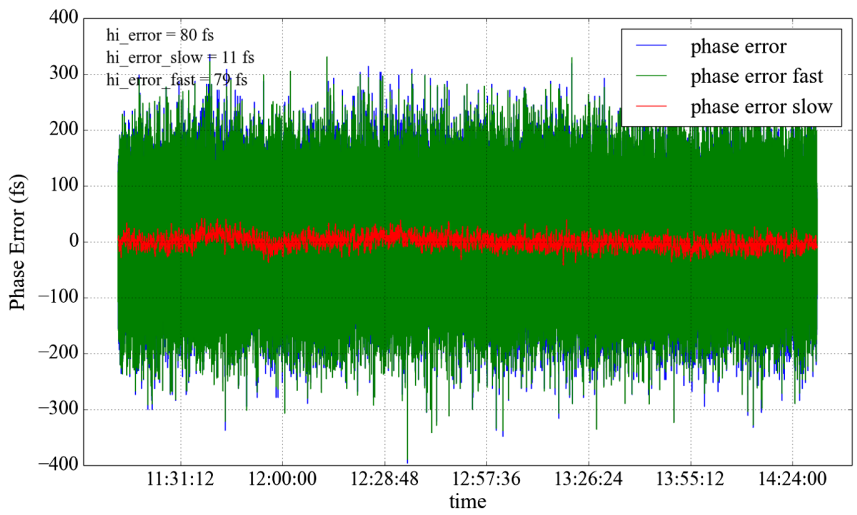

FIG. 4. The test result of the 3-hour mutual monitoring and detecting experiment: (a) the working system is running at close loop mode. (b) the monitoring system is running at open loop and detecting the phase error between the reference and driver RF from working system. 


\section{Timing and synchronization system}

The timing and synchronization system is an essential part for the XGLS. The timing jitter of the clients including the rf in the accelerator structures and the driving and scattering laser system should be less than 100 fs to generate stable electron beam and gamma-ray pulse. An integrated timing and synchronization system including the fiber based phase reference distribution system (PRDS), the digital low level rf system (LLRF) and the laser-rf synchronization client has been developed and demonstrated at Tsinghua Thomson scattering $\mathrm{x}$-ray source [33-35]. It includes one PRDS, one laser-rf synchronization client and three LLRF clients. We evaluated the PRDS with LLRF client together by taking two clients mutual monitoring and detecting experiments. The test results is shown in Fig. 4. The jitter of the working system is $54 \mathrm{fs}$ RMS monitored by itself. The jitter monitored by other independent client is 80 fs RMS. Since the phase error of monitoring system is the sum of the two independent systems, the real phase jitter of one LLRF system should be $80 / \sqrt{2}=57 \mathrm{fs}$ RMS, which is almost equal to that of working LLRF system (54 fs). The $11 \mathrm{fs}$ slow drift of the detecting system might be caused by the temperature drift between the two systems, which can be eliminated once the system works at the close loop mode. Hence, the phase jitter of the LLRF system is near 50 fs RMS.

\section{COMMISSIONING OF PHOTOCATHODE RF GUN}

The gun was baked for 72 hours under $300^{\circ} \mathrm{C}$ temperature in the vacuum furnace before installation. Nitrogen environment was employed during the installation to protect the inside surface and the cathode in the gun. After installation on the beam line, it was baked again for 48 hours under about $110-120^{\circ} \mathrm{C}$ temperature in vacuum. The gradient was increased gradually to about $110 \mathrm{MV} / \mathrm{m}$ in about 300 hours along the high power conditioning process, and finally reached about $120 \mathrm{MV} / \mathrm{m}$ in the following commissioning smoothly. There were few $\mathrm{rf}$ breakdowns occurred in the gun during the rf conditioning. The rf breakdown which limited the input power mainly occurred at the circulator and the rf window in the vacuum waveguide system. The gun was usually operated at about $108 \mathrm{MV} / \mathrm{m}$ at a repetition rate of $10 \mathrm{~Hz}$ with a rf pulse length of 1.8 us. The dark current was not measured precisely since there was no ICT or Faraday cup followed with the gun. The dark current estimated by the accumulated light intensity on YAG screen at the gun exit was about several hundred pico-Coulomb under a gradient of $108 \mathrm{MV} / \mathrm{m}$. The vacuum levels in the gun were less than $1 \times 10^{-7} \mathrm{~Pa}$ and $2.5 \times 10^{-7} \mathrm{~Pa}$ without and with the $\mathrm{rf}$ power fed into the gun, respectively.

In the previous simulation by Astra [36], a relatively low beam emittance was obtained when the launch phase

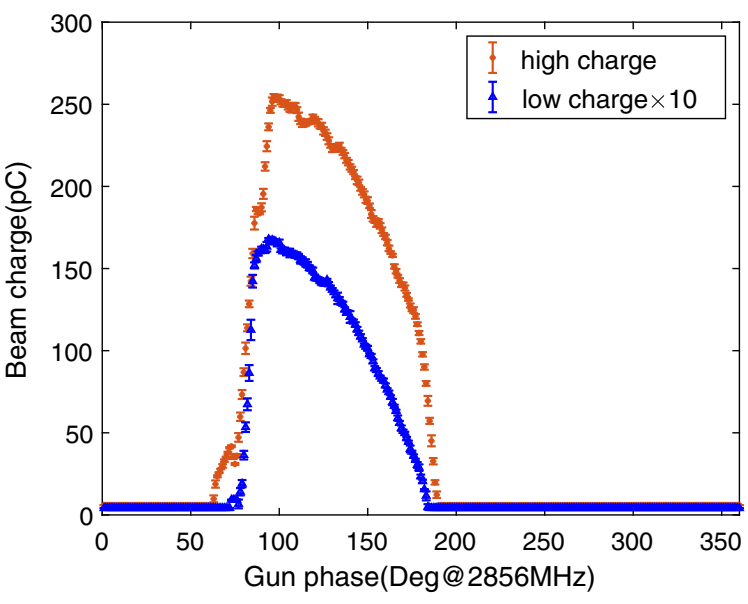

FIG. 5. Bunch charge change in gun rf phase scan. The rf phase is read from the LLRF system.

of the electron bunch center was 27 degrees from the zero crossing. A Schottky scan method was used to establish the laser launch phase in the gun. The bunch charge was measured by the SBPM and its electrics downstream of the rf gun which was carefully calibrated by the ICT installed at the exit of the first accelerator tube. The typical curves with $104 \pm 4 \mathrm{MV} / \mathrm{m}$ peak cathode field are shown in Fig. 5. The right side is close to the zero launch phase. The inflection point on the curves near 180 degrees is due to the $\sim 10$ ps driving laser pulse duration, which takes about 10 degrees in a period of S-band microwave. Based on the scan curves, the zero launch phase is defined to 185 degrees where the center of the driving laser is just at the zero crossing of the rf field at the cathode. All the following launch phases are related to zero launch phase. The scanned curves clearly demonstrate the Schottky effect in the photoemission process. The intrinsic work function can be estimated with the scan curves. According to the three-step photoemission model in metal cathodes [37], the square root of the cathode quantum efficiency

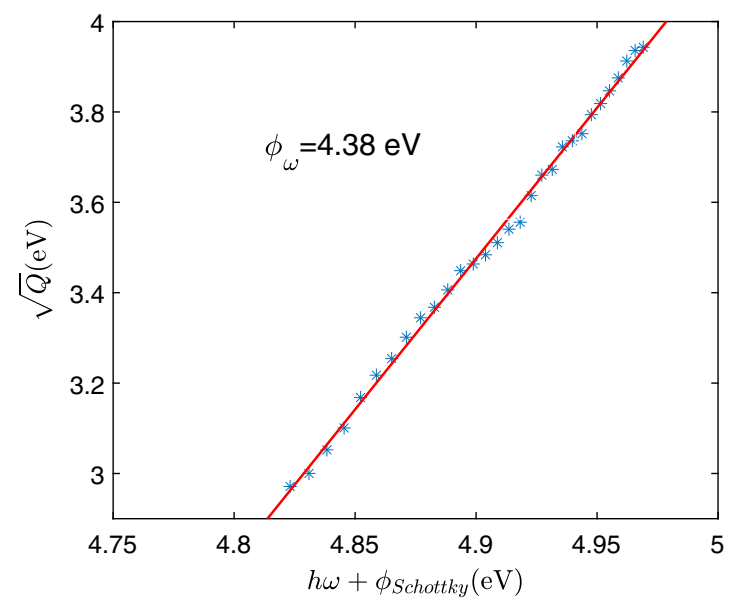

FIG. 6. An estimation of intrinsic work function by fitting the curve of $\sqrt{Q} v s\left(h \omega+\phi_{\text {Schottky }}\right)$. 

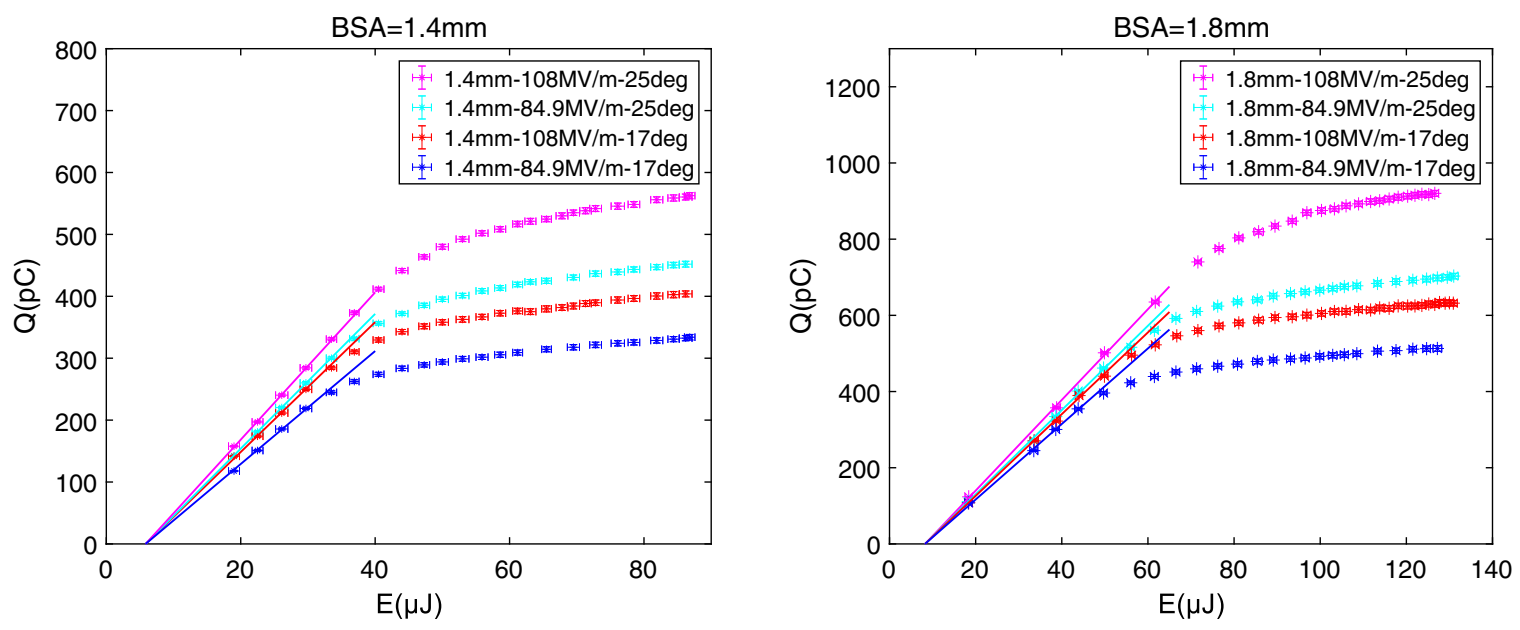

FIG. 7. Measured QE curves.

(QE) is proportional to the rest kinetic energy of the emitted electron beam, $\sqrt{\mathrm{QE}} \propto\left(h \omega+\phi_{\text {Schottky }}-\phi_{\omega}\right)$, where $h \omega$ is the photon energy, $\phi_{\text {Schottky }}$ is the Schottky work function due to Schottky effect $\phi_{\text {Schottky }}=$ $3.79 \times 10^{-2} \sqrt{E(M V / m) * \sin (\theta)} e V, \phi_{\omega}$ is the intrinsic work function of the cathode [37], $\mathrm{E}$ is the peak cathode field, and $\theta$ is the launch phase with respect to zero crossing. Since the laser energy is constant, bunch charge $\mathrm{Q}$ is linear with $\mathrm{QE}$ when the bunch charge is low and the space charge induced field makes no difference to cathode surface field and bunch charge. With the data of the low charge Schottky scan curve between 15-45 degrees of launch phase, we can fit the curve of $\sqrt{Q} v s(h \omega+$ $\left.\phi_{\text {Schottky }}\right)$ as shown in Fig. 6. The intrinsic work function of the cathode $\phi_{\omega}$ based on the fitting is $4.38 \mathrm{eV}$ and this value is close to the published work function $4.31 \mathrm{eV}$ for clean copper cathode [38,39]. That means this cathode surface is in quite good condition. When launch phase is 27 degrees and the peak cathode field is $104 \mathrm{MV} / \mathrm{m}$, taking the Schottky effect into consideration the rest kinetic energy $E_{k}$ of electron emitted from the cathode is about $0.54 \mathrm{eV}$. Knowing that the electron transverse momentum $\sigma_{\text {th }}=\sqrt{\frac{E_{k}}{3 m_{0} c^{2}}}$, we get that the thermal emittance is about $0.59 \mathrm{mmmrad} / \mathrm{mm}(\mathrm{rms})$.

In the measurement of QE, we got the change in bunch charge as a function of laser energy with two different BSA,

TABLE II. QE results.

\begin{tabular}{lll}
\hline \hline $\begin{array}{l}\text { Cathode surface } \\
\text { field }\end{array}$ & QE $(\mathrm{BSA}=1.4 \mathrm{~mm})$ & $\mathrm{QE}(\mathrm{BSA}=1.8 \mathrm{~mm})$ \\
\hline $45.6 \mathrm{MV} / \mathrm{m}$ & $(5.55 \pm 0.06) \times 10^{-5}$ & $(5.55 \pm 0.06) \times 10^{-5}$ \\
$35.9 \mathrm{MV} / \mathrm{m}$ & $(5.07 \pm 0.05) \times 10^{-5}$ & $(5.16 \pm 0.08) \times 10^{-5}$ \\
$31.6 \mathrm{MV} / \mathrm{m}$ & $(4.89 \pm 0.05) \times 10^{-5}$ & $(5.00 \pm 0.08) \times 10^{-5}$ \\
$24.8 \mathrm{MV} / \mathrm{m}$ & $(4.25 \pm 0.04) \times 10^{-5}$ & $(4.62 \pm 0.08) \times 10^{-5}$ \\
\hline \hline
\end{tabular}

$1.4 \mathrm{~mm}$ diameter and $1.8 \mathrm{~mm}$ diameter. Also we varied the peak cathode field and launch phase by changing input rf power and rf phase correspondingly. The peak cathode fields were estimated to be $84.9 \mathrm{MV} / \mathrm{m}$ and $108 \mathrm{MV} / \mathrm{m}$. The launch phases were chosen to be 17 degrees and 25 degrees from zero crossing. The measured curves are shown in Fig. 7. Intersection with the x-axis might result from some stray light mixed in the UV laser. We believe that the energy of stray light is the same when BSA diameter is the same. So we fixed the intersection with the $\mathrm{X}$-axis in the linear fit. QE is given by the slope of the straight line before the space charge force makes a difference. We get all QE results shown in Table II. The QE is measured as $5.55 \times 10^{-5}$ with a $1.8 \mathrm{~mm}$ diameter BSA at the nominal peak cathode field of $108 \mathrm{MV} / \mathrm{m}$ and launch phase of 25 degrees from the zero crossing, giving a cathode field of $45.6 \mathrm{MV} / \mathrm{m}$ during photoemission. Just as Table II shows, QE decreases due to Schottky effect as the cathode surface field becomes lower. Through above 30 minutes, we got the curve of the bunch charge as a function of time, as shown in Fig. 8. Here the sampling frequency is $5 \mathrm{~Hz}$. In the long term, the average bunch charge is $502 \mathrm{pC}$ and bunch charge jitter is $0.7 \%$.

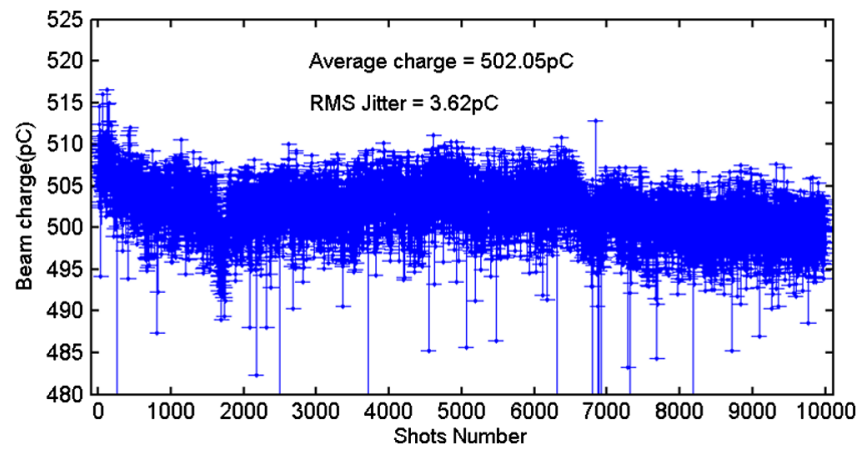

FIG. 8. Bunch charge jitter in the long term. 


\section{EMITTANCE OPTIMIZATION}

The locations of beam profile monitors are shown in Fig. 1. Transverse beam profiles are measured using six $1-\mu \mathrm{m}$ thick aluminum OTR (optical transition radiation) screens, and six 100- $\mu \mathrm{m}$ thick Ce-doped YAG (Yttrium Aluminum Garnet) screens are mounted on the same frame for low electron density use when OTR light is limited. The light from YAG and OTR screens is detected with 16-bit CCD camera. The beam profile measurement system has an imaging resolution of less than $15 \mu \mathrm{m}$. The electron bunch average energy and energy spread are measured by an analysis dipole that deflects the beam by 45 degrees. Typically, the bunch average energy is $\sim 120 \mathrm{MeV}$ and energy jitter is about $0.1 \%$ and energy spread is about $0.18 \%$. In the following emittance measurement, the bunch average energy is $103.6 \mathrm{MeV}$. Besides, we measured the bunch length with an adapted rf zero-phase crossing method. When second S-band rf cavity operates at the zero crossing of the accelerating wave, we can measure longitudinal distribution by changing the rf phase and recording beam intensity at a definite position on the OTR screen for energy measurement. The FWHM bunch length is $10.1 \mathrm{ps}$. The resolution of this method is limited by the bunch energy jitter, rf phase jitter, and energy measurement resolution. A rough estimation of bunch length measurement resolution is about 0.5 ps.

A quadrupole scan method is used in the emittance measurement [40]. As shown in Fig. 1, the second S-band tube is followed by five quadrupoles. We use the first four quadrupoles for beam matching to adjust the transverse beam size and scan the last quadrupole for emittance measurement. The OTR screen for emittance measurement is located $2.3 \mathrm{~m}$ away from the last quadrupole. Image analysis and emittance calculations are implemented by software. The software first subtracts the background determined by the average pixel value of background images. And it calculates the beamsize within the 5\% charge-cut area with two optional methods, Gaussian fit and rms. The beamsize at a certain quadrupole gradient setting is averaged with 13 images to reduce statistics error. Emittance is finally obtained by curve fitting according to the transport matrix theory. The statistics error of emittance is calculated by error transfer formula using the statistics error of beamsizes. The systematic errors mainly comes from space charge effects, the imaging optics resolution of CCD camera, the quadrupole field error and the beamenergy uncertainty. The contribution of space charge effects is estimated to be less than $0.5 \%$ by simulating the emittance measurement process with and without space charge. The imaging optics resolution is less than $15 \mu \mathrm{m}$. The quadrupole field error is about $1 \%$ and the energy uncertainty is about $1 \%$. The whole systematic error is estimated to be on the order of $10 \%$. A measurement result of 500-pC bunches as an example is shown in Fig. 9, with the beamsize calculated in the 5\% charge-cut rms method the same as all the following results. Only the statistics error is shown in the figure. In the process of emittance optimization, we also record the emittance without charge cut. From 22 sets of data, we get that a 5\% charge cut averagely reduces the emittance by about $30 \%$. Launch phase, laser spot size and gun solenoid magnet gradient are three key parameters in the injector for low emittance $[41,42]$. The laser spot size determines the thermal emittance and space charge effect that will decay the emittance. The launch phase influences the rf effect on a beam. And the gun solenoid can compensate the linear effect of space charge force on emittance if the gun solenoid magnet gradient is appropriate. In the commissioning, we systematically measured the changes in emittance of both 200-pC and 500-pC bunches with different launch phases, laser spot sizes, and gun solenoid magnets. As shown in Fig. 10, we measured both horizontal and vertical emittance of 200-pC and 500-pC bunches with a $1.8 \mathrm{~mm}$ diameter BSA. Good symmetry were observed in the emittance measurement of horizontal and vertical directions. Therefore we only cared about vertical emittance in the following measurement. For different launch phases and laser spot sizes, we need to scan gun solenoid magnet gradient to
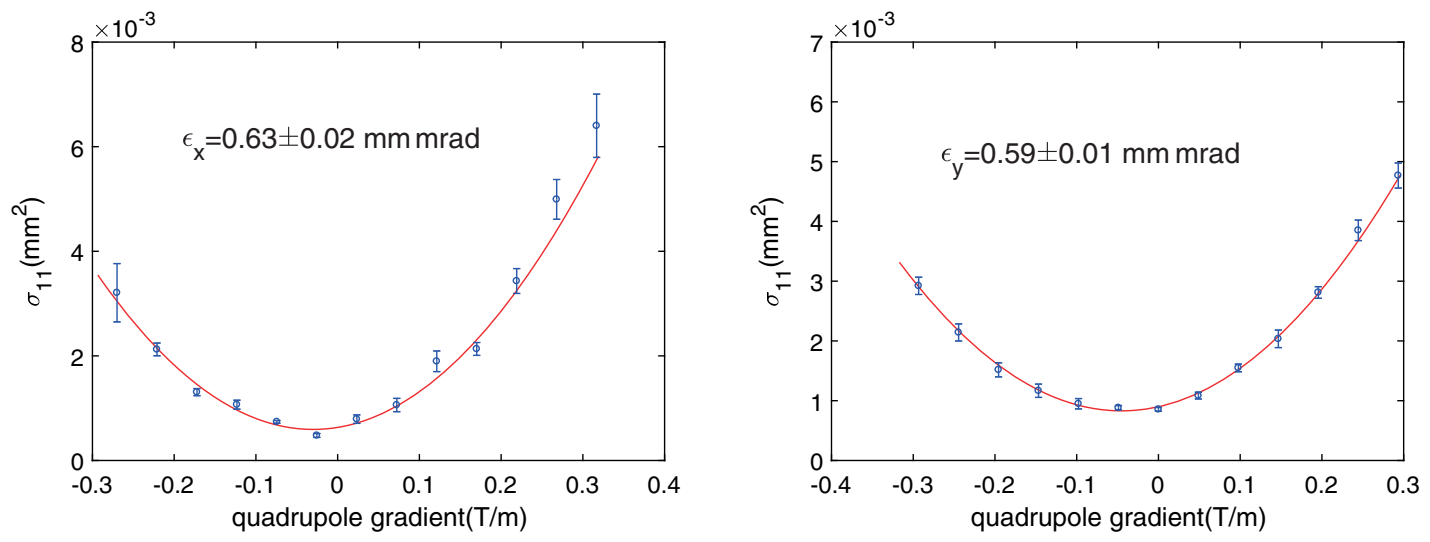

FIG. 9. A example of 500-pC bunch emittance measurement. 

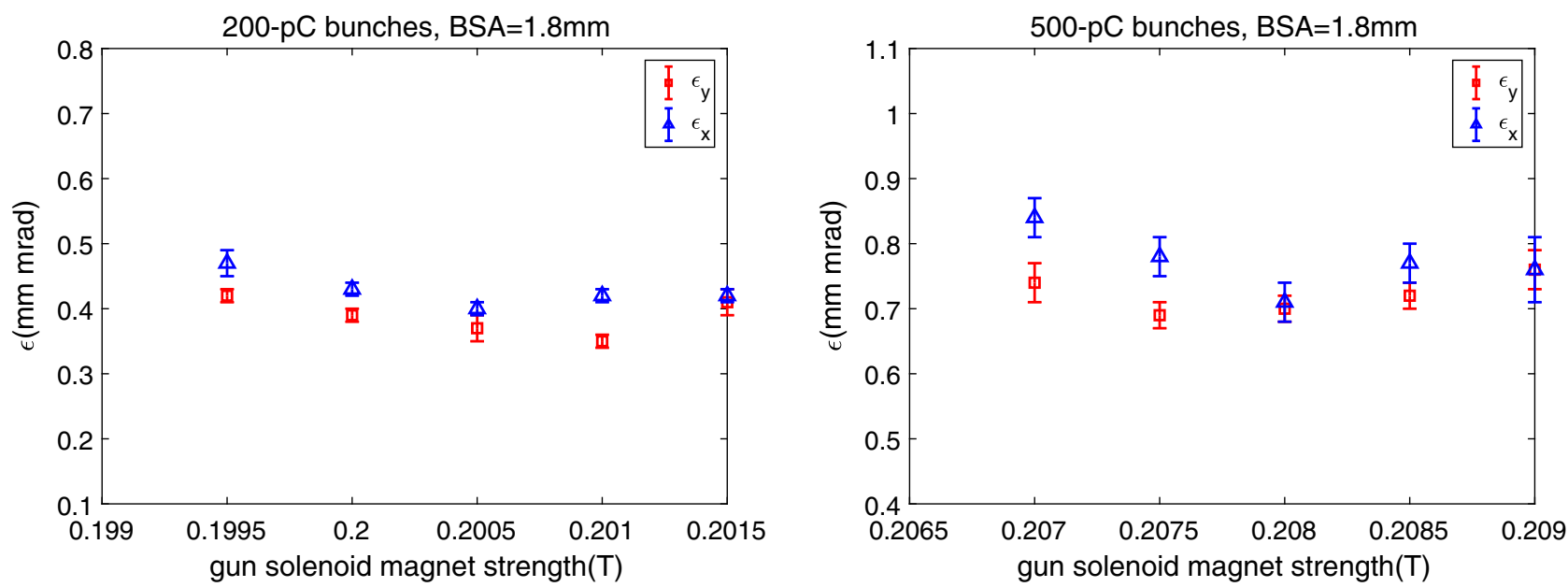

FIG. 10. Horizontal and vertical emittance of 200-pC and 500-pC bunches with a 1.8 mm diameter BSA.
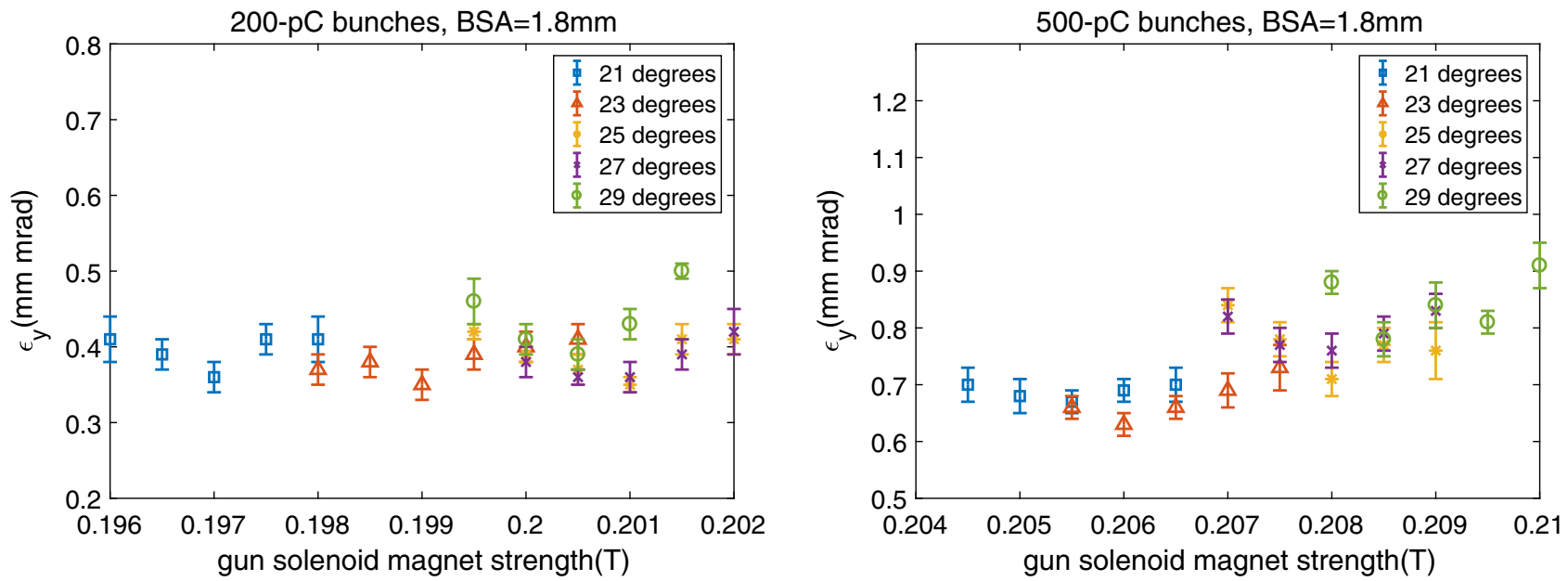

FIG. 11. The change in emittance of 200-pC and 500-pC bunches with different launch phases and 1.8 mm diameter BSA as a function of gun solenoid magnet gradient.
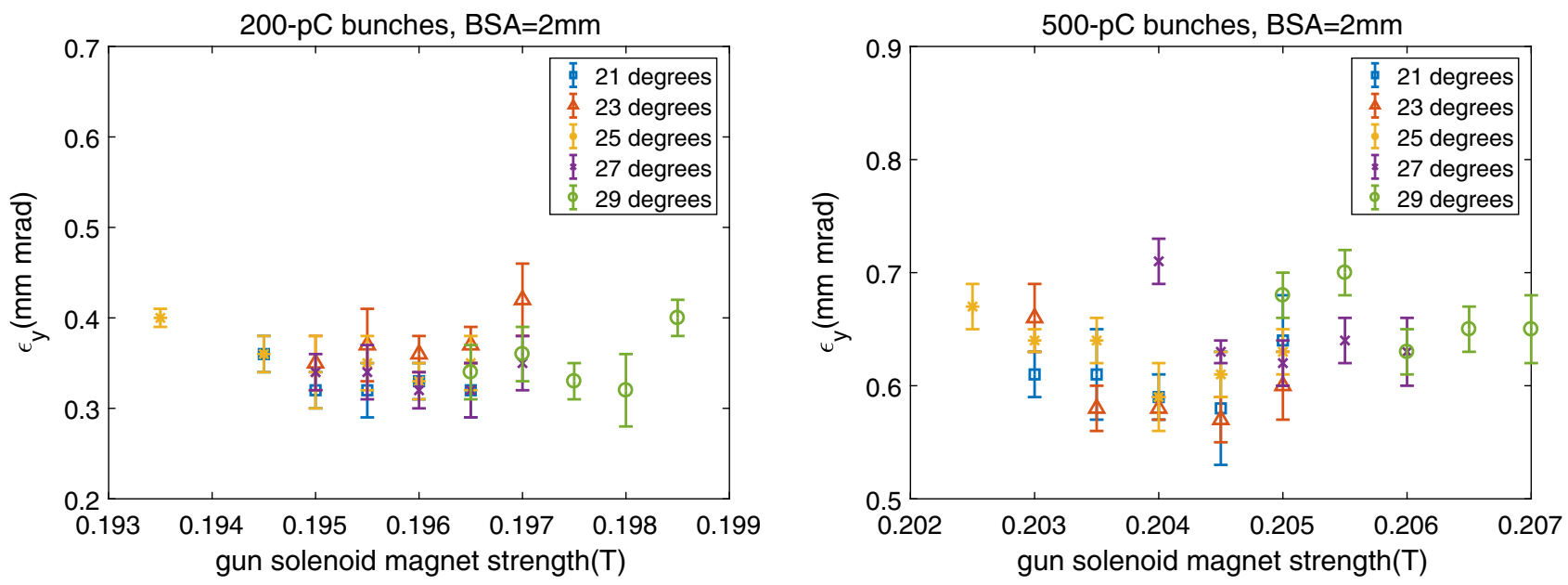

FIG. 12. The change in emittance of 200-pC and 500-pC bunches with different launch phases and 2 mm diameter BSA as a function of gun solenoid magnet gradient. 

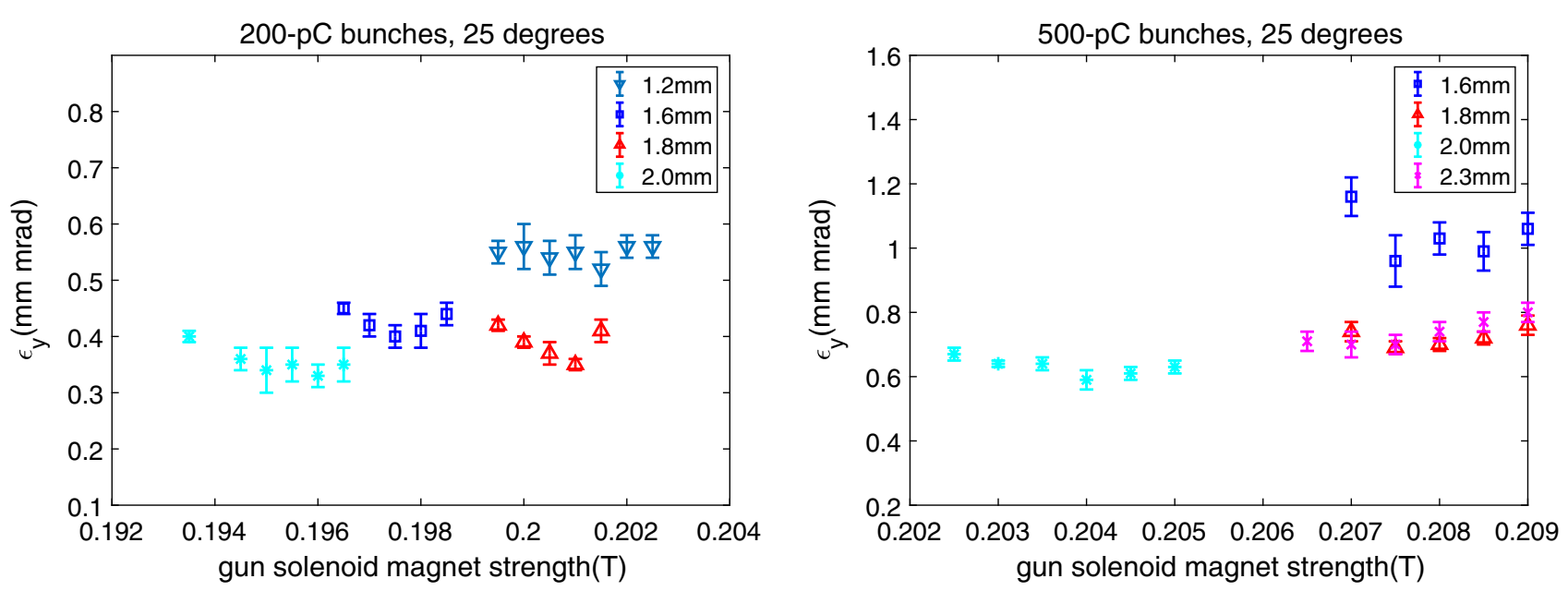

FIG. 13. The change in emittance of 200-pC and 500-pC bunches with different laser spot sizes as a function of gun solenoid magnet gradient. The launch phase is 25 degrees.

minimize emittance because emittance is quite sensitive to gun solenoid magnet gradient. We measured the change in emittance of 200-pC and 500-pC bunches with different launch phases as a function of gun solenoid magnet gradient. Figures 11 and 12 respectively correspond to a $1.8 \mathrm{~mm}$ diameter BSA and a $2 \mathrm{~mm}$ diameter BSA. For every fixed launch phase, we first scanned the gun solenoid magnet gradient to find the minimum emittance in a wide range with a relatively big step. Then we carefully measured 5 points around the minimum emittance point which is the compensated point. The compensated gun solenoid magnet gradient for 500-pC bunches is higher than that for 200-pC bunches, in agreement with previous simulations. The optimal launch phase of 500-pC bunches for both $1.8 \mathrm{~mm}$ diameter BSA and $2 \mathrm{~mm}$ diameter BSA is 23 degrees. The minimum vertical emittance of 500-pC bunches with a $2 \mathrm{~mm}$ diameter BSA is lower than $0.6 \mathrm{~mm}$ mrad. And the minimum vertical emittance of $200-\mathrm{pC}$ bunches with a $2 \mathrm{~mm}$ diameter BSA is lower than $0.35 \mathrm{~mm}$ mrad. By changing the diameter of BSA, we measured the change in emittance of 200-pC and 500-pC bunches with different laser spot sizes as a function of gun solenoid magnet gradient, which is shown in Fig. 13. The optimal laser spot size of both 200-pC bunches and 500-pC bunches is $2 \mathrm{~mm}$.

\section{SUMMARY AND FUTURE PERSPECTIVE}

In the initial system commissioning, all subsystems have operated smoothly, including drive laser system, timing and synchronization system, photocathode rf gun, beam diagnostics system and so on. Drive laser system can deliver $266 \mathrm{~nm}$ UV laser with energy jitter far less than $1 \%$ and the spatiotemporal reshaping has been done. The phase jitter of the LLRF system is near 50 fs RMS that meets the synchronization needs. The gun typically operate under a peak gradient of $108 \mathrm{MV} / \mathrm{m}$ with few rf breakdowns and a Schottky scan is used to determine the launch phase and estimate the intrinsic work function of the cathode. The intrinsic work function of the cathode is estimated to be $4.38 \mathrm{eV}$. The typical $\mathrm{QE}$ of this copper cathode is $5.55 \times 10^{-5}$. The three parameters, launch phase, laser spot size, and gun solenoid magnet gradient, are optimized to get the lowest emittance, and the minimum vertical emittance of 500-pC bunches is lower than $0.6 \mathrm{~mm}$ mrad. Then it has been demonstrated that the injector in XGLS can deliver very stable $500-\mathrm{pC}$ charge electron bunches with $0.6-0.7 \mathrm{~mm}$ mrad emittance and typically $120 \mathrm{MeV}$ energy at $10 \mathrm{~Hz}$ repetition. This lays an excellent foundation for the output of high brightness gamma-ray light. In the following phase, more accelerating tubes to increase the beam energy and a high power IR laser as the scattering laser will be installed in succession. In the future, this high intensity quasimonochromatic gamma-ray light delivered in XGLS will be used in nuclear resonance fluorescence (NRF) imaging, nuclear reaction research and so on. As for now, this injector can also be used to do research with respect to high brightness electron beam.

[1] D. Pile, First light from SACLA, Nat. Photonics 5, 456 (2011).

[2] M. Altarelli, R. Brinkmann et al., DESY Technical Design Report No. DESY-2006-097, 2006.

[3] B. Faatz, N. Baboi, V. Ayvazyan et al., Flash II: Perspectives and challenges, Nucl. Instrum. Methods Phys. Res., Sect. A 635, S2 (2011).

[4] H. S. Kang, S. J. Park et al., Current Status of PAL-XFEL Project (2013), http://accelconf.web.cern.ch/AccelConf/ IPAC2013/papers/weodb103.pdf?n=IPAC2013/papers/ weodb103.pdf.

[5] Z. Zhao, D. Wang, Q. Gu, L. Yin, M. Gu, Y. Leng, and B. Liu, Status of the SXFEL facility, Appl. Sci. 7, 607 (2017). 
[6] E. Allaria, C. Callegari, D. Cocco, W. M. Fawley, M. Kiskinova, C. Masciovecchio, and F. Parmigiani, The FERMI@Elettra free-electron-laser source for coherent X-ray physics: photon properties, beam transport system and applications, New J. Phys. 12, 075002 (2010).

[7] B. D. Patterson, R. Abela et al., Coherent science at the SwissFEL X-ray laser, New J. Phys. 12, 035012 (2010).

[8] R. Akre, D. Dowell, P. Emma et al., Commissioning the Linac Coherent Light Source injector, Phys. Rev. ST Accel. Beams 11, 030703 (2008).

[9] F. R. Elder, A. M. Gurewitsch, R. V. Langmuir, and H. C. Pollock, Radiation from electrons in a synchrotron, Phys. Rev. 71, 829 (1947).

[10] F. V. Hartemann, W. J. Brown, D. J. Gibson, S. G. Anderson, A. M. Tremaine, P. T. Springer, A. J. Wootton, E. P. Hartouni, and C. P. J. Barty, High-energy scaling of Compton scattering light sources, Phys. Rev. ST Accel. Beams 8, 100702 (2005).

[11] D. Mihalcea, B. Jacobson, A. Khizhanok, A. Murokh, P. Piot, and J. Ruan, in Proceedings of International Free Electron Laser Conference, 2017 (2018), http://lss.fnal .gov/archive/2017/conf/fermilab-conf-17-669-apc.pdf.

[12] S. Boucher, P. Frigola, A. Murokh, M. Ruelas, I. Jovanovic, J. Rosenzweig, and G. Travish, Inverse compton scattering gamma ray source, Nucl. Instrum. Methods Phys. Res., Sect. A 608, S54 (2009).

[13] F. Hartemann, S. Anderson, D. Gibson, C. Hagmann, M. Johnson, I. Jovanovic, M. Messerly, J. Pruet, M. Shverdin, A. Tremaine et al., in 2007 16th IEEE International Pulsed Power Conference (IEEE, New York, 2007).

[14] N. Ranjan, B. Terzić, G. A. Krafft, V. Petrillo, I. Drebot, and L. Serafini, Simulation of inverse Compton scattering and its implications on the scattered linewidth, Phys. Rev. Accel. Beams 21, 030701 (2018).

[15] C. Curatolo, I. Drebot, V. Petrillo, and L. Serafini, Analytical description of photon beam phase spaces in inverse Compton scattering sources, Phys. Rev. Accel. Beams 20, 080701 (2017).

[16] C. Tang, W. Huang, R. Li et al., Tsinghua Thomson scattering X-ray source, Nucl. Instrum. Methods Phys. Res., Sect. A 608, S70 (2009).

[17] Z. Zhao, S. Chen, L. Yu, C. Tang, L. Yin, D. Wang, Q. Gu et al., in Proceedings of the 2nd International Particle Accelerator Conference, San Sebastiáán, Spain (EPS-AG, Spain, 2011), pp. 3011-3013.

[18] M. Zhang, D. Gu, Q. Gu, D. Huang, and Z. Wang, in Proceedings of IPAC2017, Copenhagen, Denmark, 2017 (JACoW, Geneva, Switzerland, 2017), https://accelconf .web.cern.ch/AccelConf/ipac2017/papers/tupab083.pdf.

[19] P. Zhu, Y. Zhu, Y. Hidaka, L. Wu, J. Cao, H. Berger, J. Geck, R. Kraus, S. Pjerov, Y. Shen et al., Femtosecond time-resolved $\mathrm{MeV}$ electron diffraction, New J. Phys. 17, 063004 (2015).

[20] D. T. Palmer, R. H. Miller, H. Winick, X. J. Wang, K. Batchelor, M. H. Woodle, and I. Ben-Zvi, Microwave measurements and beam dynamics simulations of the BNL/SLAC/UCLA emittance-compensated 1.6-cell photocathode rf gun, in Electron-Beam Sources and ChargedParticle Optics (International Society for Optics and Photonics, San Diego, CA, USA, 1995).
[21] K. T. McDonald, Design of the laser-driven RF electron gun for the BNL accelerator test facility, IEEE Trans. Electron Devices 35, 2052 (1988).

[22] H. Qian, H. Chen, Y. Du, W. Huang, C. Li, X. Liu, X. Lu, C. Tang et al., in Proceedings of the 2nd International Particle Accelerator Conference, San Sebastiáán, Spain (EPS-AG, Spain, 2011).

[23] L. Zheng, Y. Du, Z. Zhang et al., Development of S-band photocathode RF guns at Tsinghua University, Nucl. Instrum. Methods Phys. Res., Sect. A 834, 98 (2016).

[24] F. Stephan and M. Krasilnikov, High brightness photo injectors for brilliant light sources, Synchrotron Light Sources and Free-Electron Lasers: Accelerator Physics, Instrumentation and Science Applications (Springer, Switzerland, 2016), pp. 1-38.

[25] J. Yang, F. Sakai, T. Yanagida, M. Yorozu, Y. Okada, K. Takasago, A. Endo, A. Yada, and M. Washio, Lowemittance electron-beam generation with laser pulse shaping in photocathode radio-frequency gun, J. Appl. Phys. 92, 1608 (2002).

[26] A. Brachmann, R. Coffee, D. Dowell et al., http://citeseerx .ist.psu.edu/viewdoc/download?doi=10.1.1.624.8254\&rep= rep1\&type=pdf.

[27] M. Quattromini, L. Giannessi, C. Ronsivalle, and C. Enea, in Proceedings of FEL2004 Conference (Comitato Conferenze Elettra, Trieste, Italy, 2004).

[28] I. Will and G. Klemz, Generation of flat-top picosecond pulses by coherent pulse stacking in a multicrystal birefringent filter, Opt. Express 16, 14922 (2008).

[29] L. Yan, J. Hua, Y. Du, Y. Huang, Y. You, D. Wang, W. Huang, and C. Tang, UV pulse trains by $\alpha$-BBO crystal stacking for the production of THz-rap-rate electron bunches, J. Plasma Phys. 78, 429 (2012).

[30] W. Plaß, H. Rottke, W. Heuer, G. Eichhorn, and H. Zacharias, Surface sum-frequency mixing for auto- and cross-correlation of ultrashort UV and IR pulses, Appl. Phys. B 54, 199 (1992).

[31] I. Will, H. I. Templin, S. Schreiber, and W. Sandner, Photoinjector drive laser of the FLASH FEL, Opt. Express 19, 23770 (2011).

[32] J. T. Hunt, J. A. Glaze, W. W. Simmons, and P. A. Renard, Suppression of self-focusing through low-pass spatial filtering and relay imaging, Appl. Optics 17, 2053 (1978).

[33] Z. Lin, Y. Du, J. Yang, Y. Xu, L. Yan, W. Huang, C. Tang, G. Huang, Q. Du, L. Doolittle et al., Development of sub-100 femtosecond timing and synchronization system, Rev. Sci. Instrum. 89, 014701 (2018).

[34] J. Yang, Y. Du, L. Yan et al., Laser-RF synchronization based on digital phase detector, Nucl. Sci. Tech. 28, 57 (2017).

[35] Z. Lin, J. Byrd, L. Doolittle, Y.-C. Du, G. Huang, W. Huang, D. Qiang, C. Tang, R. Wilcox, Y. Xu et al., in IPAC17 (2017), https://accelconf.web.cern.ch/AccelConf/ ipac2017/papers/thpva055.pdf.

[36] K. Flöttmann, Astra dokumentation, 2013, http://www .desy.de/ mpyflo/.

[37] D. H. Dowell and J. F. Schmerge, Quantum efficiency and thermal emittance of metal photocathodes, Phys. Rev. ST Accel. Beams 12, 1 (2009). 
[38] D. H. Dowell, F. K. King et al., http://inspirehep.net/ record/695678/files/slac-pub-11455.pdf.

[39] C. P. Hauri, R. Ganter, F. Le Pimpec, A. Trisorio, C. Ruchert, and H. H. Braun, Intrinsic Emittance Reduction of an Electron Beam from Metal Photocathodes, Phys. Rev. Lett. 104, 234802 (2010).

[40] H. Lee, S. Karkare, L. Cultrera, A. Kim, and I. V. Bazarov, Review and demonstration of ultra-low-emittance photocathode measurements, Rev. Sci. Instrum. 86, 073309 (2015).
[41] M. Krasilnikov, F. Stephan, G. Asova, H.-J. Grabosch, M. Groß, L. Hakobyan, I. Isaev, Y. Ivanisenko, L. Jachmann, M. Khojoyan et al., Experimentally minimized beam emittance from an $L$-band photoinjector, Phys. Rev. ST Accel. Beams 15, 100701 (2012).

[42] D. H. Dowell, Slac, https://arxiv.org/ftp/arxiv/papers/1610/ 1610.01242.pdf. 